\title{
ANÁLISE ECONOMÉTRICA DA PRODUÇÃO DE MADEIRA SERRADA NO BRASIL
}

\author{
Renato Vinícius Oliveira Castro ${ }^{1}$, Ana Flávia Neves Mendes ${ }^{2}$, Glauciana da Mata Ataíde ${ }^{1}$, \\ Carlos Alberto Araújo Júnior ${ }^{1}$, Gustavo Eduardo Marcatti ${ }^{1}$, Daniel de Paula Silveira ${ }^{1}$, Camila Brás Costa ${ }^{2}$ \\ ${ }^{1}$ Eng. Florestal, Pós-graduando em Ciência Florestal, UFV, Viçosa, MG, Brasil - castrorvo@ymail.com; carlosmuc@hotmail.com; \\ gustavomarcatti@gmail.com; daniel.silveira@ufv.br; \\ ${ }^{2}$ Eng $^{\mathrm{a}}$. Florestal, Pós-graduanda em Ciência Florestal,UFV, Viçosa, MG, Brasil - glaucianadamata @yahoo.com.br; mila_ufv@yahoo.com.br
}

Recebido para publicação: 10/11/2011 - Aceito para publicação: 08/11/2012

\begin{abstract}
Resumo
Estudos que objetivam analisar o mercado madeira serrada no Brasil merecem destaque por fornecerem orientações sobre sua tendência. Após o avanço no uso de painéis de madeira, o mercado sofreu influências por esse substituto, se fazendo necessárias análises econométricas para dimensionar e planejar a produção, diminuindo riscos e incertezas de comercialização. Esse mercado é dividido em madeiras de não coníferas e coníferas, que contribuem atualmente com $62 \%$ e $38 \%$ do mercado, respectivamente. Com base em uma série temporal anual da produção brasileira de madeira serrada de não coníferas e coníferas no período de 1961 a 2009, publicada pela Food and Agriculture Organization (FAO, 2011), o presente trabalho objetivou avaliar a metodologia Box \& Jenkins (BOX; JENKINS, 1976) para realizar previsões da produção desse mercado. Os modelos foram avaliados com base nos critérios de Akaike e Schwarz, na significância dos coeficientes, no princípio de parcimônia e no comportamento dos resíduos. Pelos resultados, conclui-se que o modelo autorregressivo de média móvel (ARIMA) $(2,1,1)$ foi adequado para prever a produção de madeira serrada de não coníferas, e o modelo $\operatorname{ARIMA}(1,1,1)$ para prever a produção de madeira serrada de coníferas. A metodologia pode ser utilizada para previsão desse mercado.
\end{abstract}

Palavras-chave: Produção de madeira serrada; séries temporais; metodologia Box \& Jenkins.

\begin{abstract}
Econometric analysis of sawn timber production in Brazil. Studies that aim to analyze the lumber market in Brazil are noteworthy for providing guidance on its trend. After advance in wood panels using the market has been influenced by this replacement, and it was necessary an econometric analysis to scale and plan production, reducing risks and uncertainties. Such market is divided into non-coniferous woods and conifers, which currently contribute to $62 \%$ and $38 \%$ of the market, respectively. Based on an annual time series of non-coniferous and coniferous sawn timber Brazilian production in the 1960 to 2009 period, published by the Food and Agriculture Organization (FAO, 2011), the present research aimed to evaluate the methodology Box \& Jenkins (BOX; JENKINS, 1976) to forecast the production of this market. The models were evaluated based on the Akaike and Schwarz criteria, at the coefficients significance, at the parsimony principle and at the waste behavior. According to results, the moving average auto-regressive model (ARIMA) $(2,1,1)$ was adequate to predict the non-coniferous sawn timber production and the ARIMA $(1,1,1)$ model to predict the conifers sawn wood production. The methodology can be used for such market prediction. Keywords: Lumber production; time series; Box \& Jenkins methodology.
\end{abstract}

\section{INTRODUÇÃO}

A madeira é um material utilizado pelo homem desde os tempos pré-históricos, para obtenção de energia, confecção de utensílios e abrigos, entre outros usos, e até os dias atuais desempenha um papel muito importante em vários segmentos econômicos, como o mobiliário e a construção civil (ZENID, 1997; CASTRO, 2011). Madeira serrada é uma denominação genérica dada à utilização dos vários formatos e dimensões das peças de madeira para a produção de produtos como pranchas, blocos, tábuas, dormentes, perfis e vigas, dentre outros, após o desdobro da madeira (ABIMCI, 2011). 
Segundo Perez e Bacha (2006), os produtos elaborados pela indústria de madeira serrada no Brasil são produzidos com a utilização de madeiras provenientes de não coníferas, como o mogno, o cedro e o eucalipto, e de madeiras originadas de coníferas, como a araucária e o pínus. A madeira das folhosas (não coníferas) origina-se quase que totalmente da floresta amazônica, enquanto as coníferas são produzidas principalmente no sul do país, nas florestas plantadas de Pinus (PONCE, 1995). De acordo com Vital (2009), a floresta amazônica é responsável por 85\% da produção de madeira nativa no Brasil, onde há grande concentração de serrarias.

O mercado de madeira serrada do Brasil ocupou a $3^{a}$ posição no ranking mundial de produção (FAO, 2011), com produção aproximada de 25 milhões $\mathrm{m}^{3}$ em 2009, a qual apresentou pouca variação em relação ao ano anterior. $\mathrm{O}$ surgimento de produtos substitutos à madeira serrada, como os painéis reconstituídos de madeira para a indústria moveleira e a utilização de vidro, aço, alumínio e ligas no mobiliário, aliado à pressão ambiental decorrente dos problemas ambientais da exploração predatória da floresta amazônica, colocam em risco o crescimento desse mercado.

Nesse cenário de transição, é de fundamental importância o desenvolvimento de pesquisas relacionadas ao comportamento futuro do mercado de madeira serrada brasileiro, de forma a contribuir para fornecer orientações aos produtores sobre os melhores meios de selecionar seus empreendimentos, dimensionar suas vendas e planejar o volume de estoques (CORDEIRO et al., 2010).

A análise econométrica investiga as respostas, em curto prazo, do sistema econômico às diversas mudanças a ele impostas, das mais variadas formas (CASTRO et al., 2011). Essas análises têm se tornado cada dia mais importantes na economia moderna, e, considerando a importância do setor florestal, a falta de consenso sobre o comportamento da oferta e demanda de madeira e as raras informações providas de caráter quantitativo, estudos econométricos para o referido mercado devem ser realizados (ALMEIDA et al., 2009).

Nesse contexto, o objetivo do presente trabalho foi avaliar a metodologia Box \& Jenkins, empregada para a realização de previsões de séries temporais, para subsidiar o planejamento e dimensionamento da produção de madeira serrada (origem de florestas plantadas e naturais) de coníferas e não coníferas no Brasil.

\section{MATERIAL E MÉTODOS}

Os dados utilizados são provenientes de séries temporais anuais da produção de madeira serrada de não coníferas e coníferas no Brasil (origem de florestas plantadas e naturais), publicados pela Food and Agriculture Organization (FAO, 2011). O período disponibilizado compreende os anos entre 1961 e 2009. Para ajuste dos modelos, considerou-se o período de 1961 a 2005.

Foram deixadas de fora as quatro últimas observações, conforme Castro et al. (2011), com o objetivo de validar o modelo, a partir da comparação dos dados reais com os previstos, tanto para a produção brasileira de madeira de não conífera quanto para a madeira de conífera.

Para o estudo de projeção, foi usado o modelo autorregressivo integrado de média-móvel, conhecido como metodologia de Box \& Jenkins (BOX; JENKINS, 1976).

Segundo Castro et al. (2011), para aplicação da metodologia de Box \& Jenkins, as séries temporais necessitam ser estacionárias ou passíveis de estacionariedade. Essa característica é fundamental para a previsão com base na regressão de séries temporais, solidificando a premissa de que o futuro se comportará de acordo com o passado (GUJARATI, 2006). Segundo esse mesmo autor, para uma série de dados ser estacionária, suas variáveis não podem apresentar tendências e devem ser estáveis ao longo do tempo.

Inicialmente, para verificação de estacionariedade, foi realizada uma análise gráfica das séries e aplicado o teste de raiz unitária (Teste ADF) (SEDDIGHI et al., 2000). Se a série analisada não for estacionária em nível (na unidade original), devem-se realizar transformações nos dados (GUJARATI, 2006) até que a condição de estacionariedade se torne válida (CORDEIRO et al., 2010).

Depois de obtida a série estacionária, aplica-se a metodologia de Box \& Jenkins com segurança nas previsões (MYNBAEV; LEMOS, 2004).

A metodologia Box \& Jenkins consiste em quatro etapas, a saber: identificação, estimação, verificação e previsão.

A identificação consiste em descobrir qual dentre as várias versões dos modelos de Box \& Jenkins descreve o comportamento da série. A identificação do modelo a ser estimado ocorre pelo comportamento das funções de autocorrelações (FAC) e das funções de autocorrelações parciais (FACP). 
Segundo Rocha (2003), citado por Cordeiro (2010), tem-se as seguintes classes de modelos:

1. Média Móvel (MA): No modelo MA, a série temporal $Y_{t}$ é resultado da combinação linear dos termos aleatórios em $t$ e em períodos anteriores. Em sua forma geral, um $\operatorname{MA}(q)$, leva em consideração $q$ valores defasados de $\varepsilon: Y_{t}=\varepsilon_{t}-\theta_{1} \varepsilon_{t-1}-\theta_{2} \varepsilon_{t-2} \ldots \ldots . . . \theta_{p} \varepsilon_{t-p}$, em que $\theta=$ parâmetro; e $\varepsilon_{t}=$ termos aleatórios.

2. Autorregressivo $(A R)$ : No modelo $\mathrm{AR}$, a série temporal $Y_{t}$ é descrita somente pelos seus valores realizados e pelos termos aleatórios. O referido modelo de ordem $p \operatorname{AR}(p)$ será o resultado da soma ponderada de seus $p$ valores passados e dos termos aleatórios: $Y_{t}=\phi_{1} Y_{t-1}+\phi_{2} Y_{t-2}+\ldots \ldots \ldots+\phi_{p} Y_{t-p}+\varepsilon_{t}$, em que: $\phi_{t}=$ parâmetro; e $\varepsilon_{t}=$ termos aleatórios.

3. Média Móvel Autorregressivo (ARMA): No modelo ARMA, a série temporal $Y_{t}$ é função de seus valores históricos e pelos termos aleatórios corrente e passados. Generalizando, esse modelo é representado como: $Y_{t}=\phi_{1} Y_{t-1}+\ldots \ldots .+\phi_{p} Y_{t-p}+\varepsilon_{t}-\theta_{1} \varepsilon_{t-1}-\ldots \ldots .-\theta_{p} \varepsilon_{t-p}$.

4. Média Móvel, Integrado e Autorregressivo (ARIMA): Se a série temporal analisada não for estacionária em nível, devem-se aplicar diferenças até que a condição de estacionariedade se torne válida. O número de diferenças necessárias é denominado ordem de integração (I). Sendo $Y_{t}$ estacionária após ser diferenciada $d$ vezes, com a série derivada desse processo podendo ser descrita como um modelo $\operatorname{ARMA}(p, q)$, diz-se que a série temporal $Y_{t}$ segue um modelo $\operatorname{ARIMA}(p, d, q)$ :

$$
W_{t}=\phi_{1} W_{t-1}+\ldots \ldots+\phi_{p} W_{t-p}+\varepsilon_{t}-\theta_{1} \varepsilon_{t-1}-\ldots . .-\theta_{q} \varepsilon_{t-q}, \text { em que: } W_{t}=\Delta^{d} Y_{t} .
$$

Os correlogramas, que são as representações gráficas das FACs e FACPs, indicam quais as ordens de AR e MA utilizar (GUJARATI, 2006; ENDERS, 1995). Se um lag de autocorrelação estiver fora do intervalo de confiança, torna-se necessária a inclusão de um parâmetro para esse lag.

Empregou-se o teste de Dickey-Fuller Aumentado (ADF), que pode ser aplicado nas seguintes formas, conforme Seddighi et al. (2000): sem constante e sem tendência: $\Delta X_{t}=\delta X_{t-1}+\sum_{i=1}^{m} \delta_{i} \Delta X_{t-i}+u_{t}$; com constante e sem tendência: $\Delta X_{t}=\beta_{1}+\delta X_{t-1}+\sum_{i=1}^{m} \delta_{i} \Delta X_{t-i}+u_{t}$; ou com constante e com tendência: $\Delta X_{t}=\beta_{1}+\beta_{2} T+\delta X_{t-1}+\sum_{i=1}^{m} \delta_{i} \Delta X_{t-i}+u_{t} ; \quad$ em que $\beta=$ constante; $T=$ parâmetro de tendência; $\delta$ = parâmetros associados às ordens de AR e MA.

$\mathrm{Na}$ etapa de estimação, foram obtidos os parâmetros dos componentes autorregressivos e dos componentes de médias móveis, previamente selecionados.

A verificação consiste em checar se o modelo escolhido é adequado para os fins desejados. Para tanto, verifica-se o comportamento dos resíduos do modelo estimado. Para que o modelo estimado seja considerado adequado, é necessário que os seus resíduos sejam estimativas de ruído branco, ou seja, devem se comportar de maneira aleatória e devem ser não significativos (GUJARATI, 2006).

Quando se obtém um modelo satisfatório, passa-se para a última etapa da metodologia, que constitui o objetivo principal: realizar previsões.

Depois de estimados e avaliados, foram realizadas quatro previsões para cada modelo escolhido, para validar o poder preditivo dos mesmos. Toda a análise foi realizada com auxílio do software Eviews 5.0 (ECONOMETRIC EVIEWS, 2004).

\section{RESULTADOS E DISCUSSÃO}

\section{Estacionariedade}

Pela análise da figura 1a, verifica-se a presença de tendência crescente ao longo das séries de produção de madeira de não coníferas e coníferas, o que revela que as séries não são estacionárias. Ao se aplicarem as primeiras diferenças (Figura 1b), em ambas as séries, aparentemente passaram a se comportar de forma estacionária (sem tendência).

Para verificar estatisticamente a estacionariedade das séries, aplicou-se o teste ADF a $1 \%$ de significância (Tabela 1). 
(a)

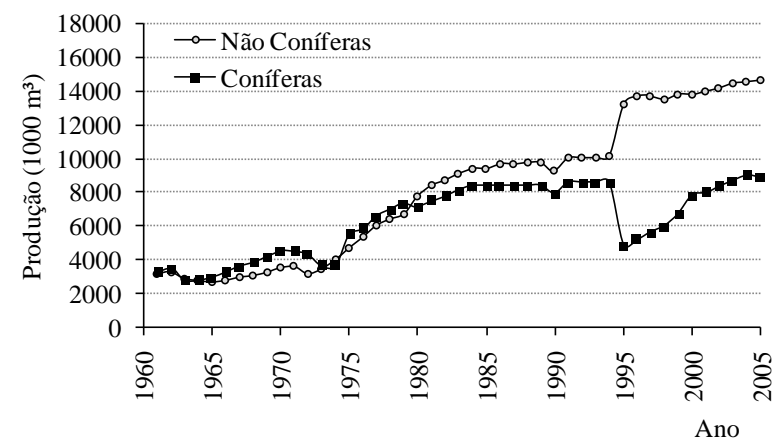

(b)

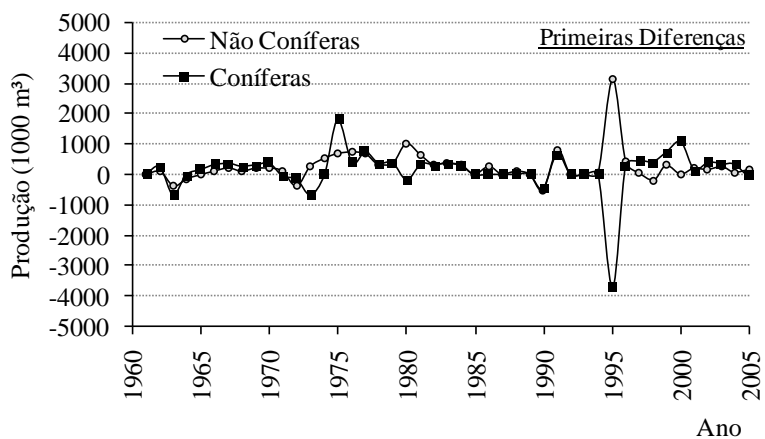

Figura 1. Séries de produção brasileira de madeira serrada de não coníferas e coníferas, em nível (a) e em primeiras diferenças (b).

Figure 1. Brazilian production series of lumber from non-coniferous and coniferous trees, in level (a) and in first differences (b).

Tabela 1. Resultados do teste de ADF em nível e em primeiras diferenças para as séries anuais de produção de madeira serrada de não coníferas e coníferas no Brasil, de 1961 a 2005.

Table 1. Results of the ADF test in level and in first differences for the annual production series of nonconiferous lumber and coniferous in Brazil, from 1961 to 2005.

\begin{tabular}{lcccc}
\hline Madeira & & Modelo & Teste ADF & Probabilidade \\
\hline Não conífera & Nível & Sem tendência e sem constante & 2,9807 & 0,9990 \\
& Nível & Somente com constante & 0,0411 & 0,9575 \\
& Nível & Com tendência e constante & $-2,6740$ & 0,2515 \\
& Primeiras diferenças & Sem tendência e sem constante & $-5,0740$ & 0,0000 \\
\hline Conífera & Nível & Sem tendência e sem constante & 0,8485 & 0,8905 \\
& Nível & Somente com constante & $-1,2173$ & 0,6596 \\
& Nível & Com tendência e constante & $-1,9026$ & 0,6377 \\
& Primeiras diferenças & Sem tendência e sem constante & $-6,1597$ & 0,0000 \\
\hline
\end{tabular}

Os resultados do teste $\mathrm{ADF}$ confirmam que as séries de produção sob análise não são estacionárias em nível. $\mathrm{O}$ valor de $\mathrm{p}$ (probabilidade) $>0,01$ indica a não estacionariedade da série. Ao se aplicar o teste nas primeiras diferenças, as séries de produção de madeira tornaram-se estacionárias $(\mathrm{p}<0,01)$ na categoria sem tendência e sem constante.

\section{Identificação}

Para a série de produção de madeira serrada de não coníferas, em primeiras diferenças, observaram-se, na FAC, picos nos lags 1, 4, 5 e 10, indicando a inclusão dos MA(1), MA(4), MA(5) e MA(10). Na FACP, picos nos lags 1 e 12, indicando a inclusão dos AR(1) e AR(12) (Figura 2).

\section{Estimação}

Para a seleção dos modelos, foram considerados os lags indicados pelos correlogramas da FAC e da FACP, o princípio de parcimônia, a significância dos coeficientes, o comportamento dos resíduos e os critérios de informação de Akaike (AIC) e Schwarz (SCH). Foram selecionados os modelos com o menor número de coeficientes significativos e com menores valores para os critérios AIC e SCH.

Vários modelos foram estimados para o período de 1961 a 2005 para as duas séries de produção. As equações 1, 2 e 3 para previsão da série de produção de madeira de não coníferas apresentaram coeficientes não significativos, que foram eliminados dos modelos, resultando na equação 4 , que foi selecionada, sendo um modelo ARIMA $(2,1,1)$, com dois parâmetros autorregressivos integrados em primeiras diferenças e com um parâmetro de média móvel. Os valores de AIC e SCH mantiveram-se estáveis com a exclusão dos parâmetros não significativos. 
(a)

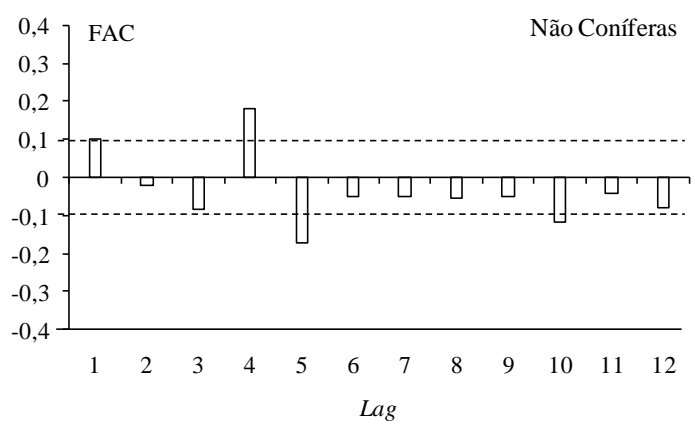

(c)

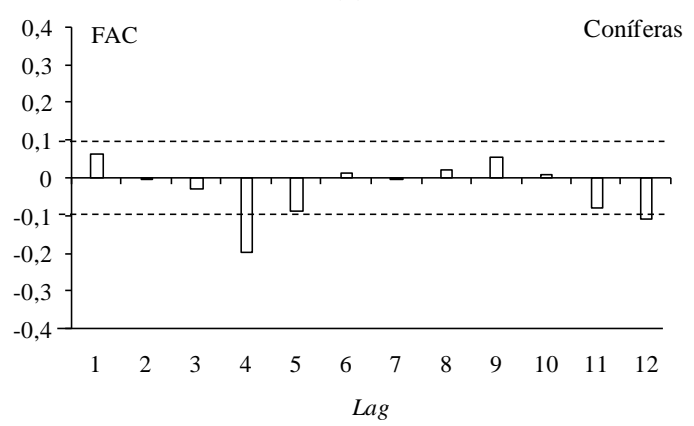

(b)

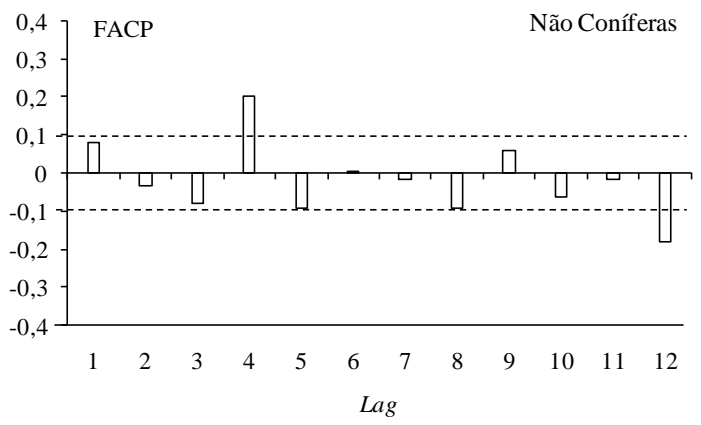

(d)

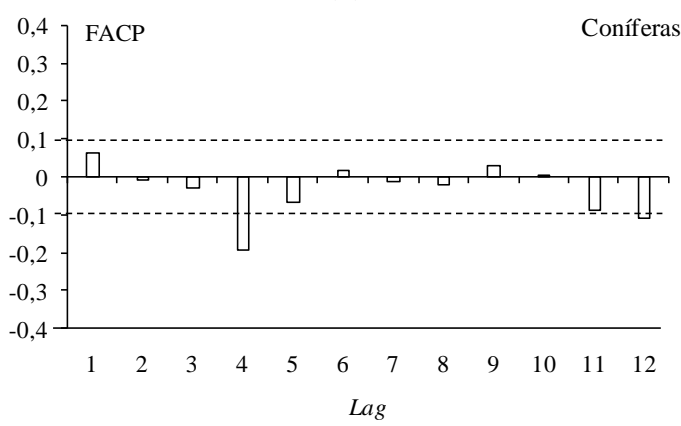

Figura 2. FAC (a) e FACP (b) em primeiras diferenças para a série de madeira de não coníferas e de coníferas (c e d), 1961 a 2009.

Figure 2. FAC (a) and FACP (b) in first differences for the non-coniferous and coniferous wood series (c and d), from 1961 to 2009.

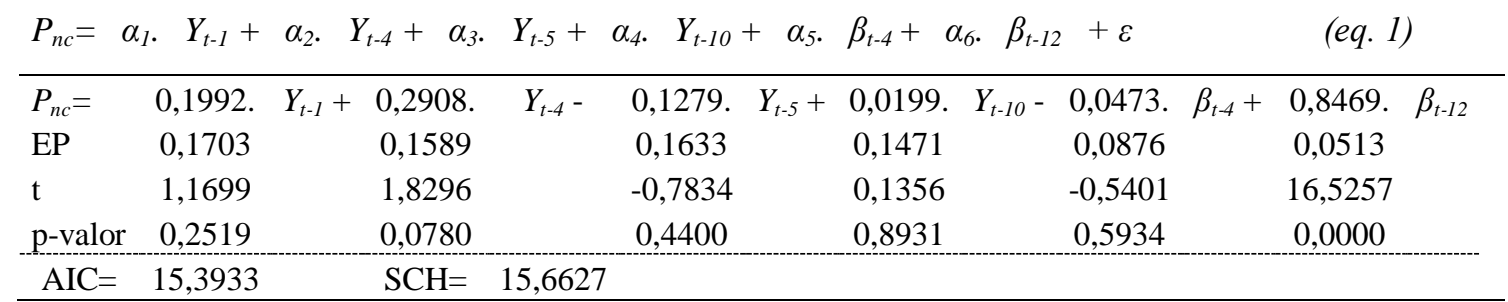

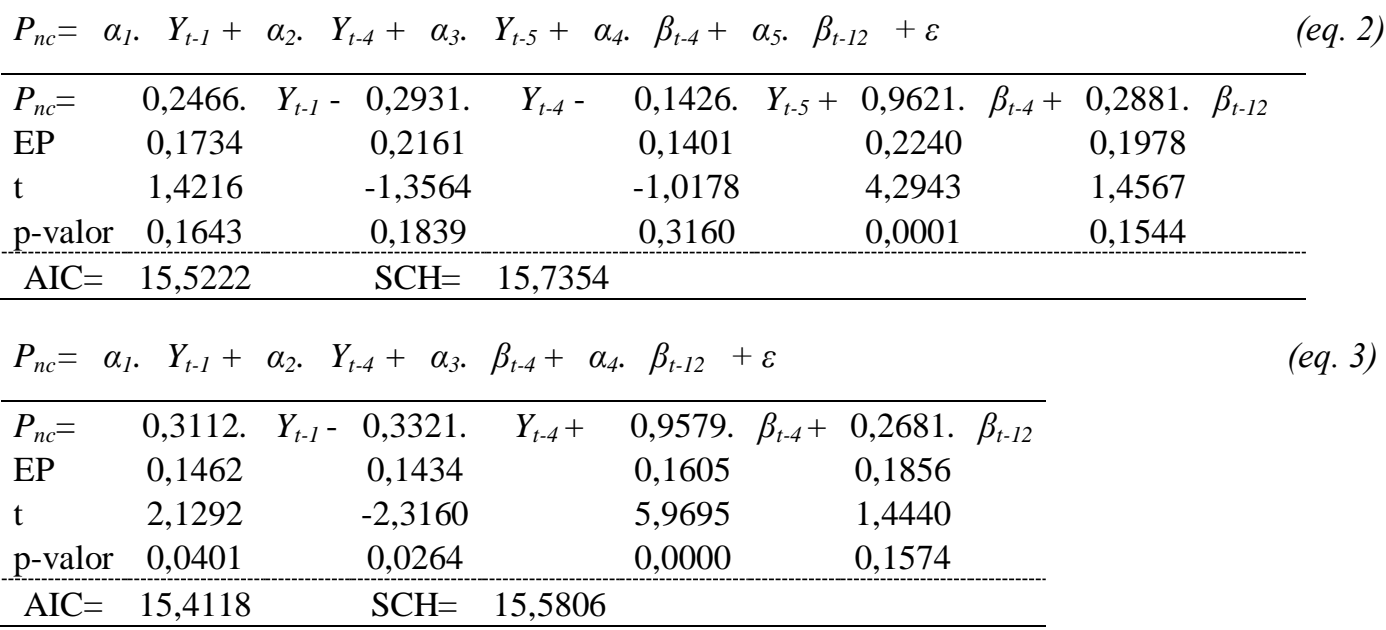




\begin{tabular}{|c|c|c|c|c|}
\hline$P_{n c}=$ & 0,3386 & $Y_{t-1}-0,390117$ & $Y_{t-4}+$ & $0,890792 . \quad \beta_{t-4}$ \\
\hline EP & 0,1420 & 0,1703 & & 0,0519 \\
\hline $\mathrm{t}$ & 2,3850 & $-2,2904$ & & 17,1519 \\
\hline p-valor & 0,0223 & 0,0278 & & 0,0000 \\
\hline $\mathrm{AIC}=$ & 15,5379 & $\mathrm{SCH}=$ & 15,6646 & \\
\hline
\end{tabular}

(eq. 4)

As equações 5 e 6 para previsão da série de produção de madeira de coníferas apresentaram coeficientes não significativos, e a selecionada é representada na equação 7 , sendo um ARIMA $(1,1,1)$. Os valores de AIC e SCH também se mantiveram estáveis com a exclusão dos parâmetros não significativos.

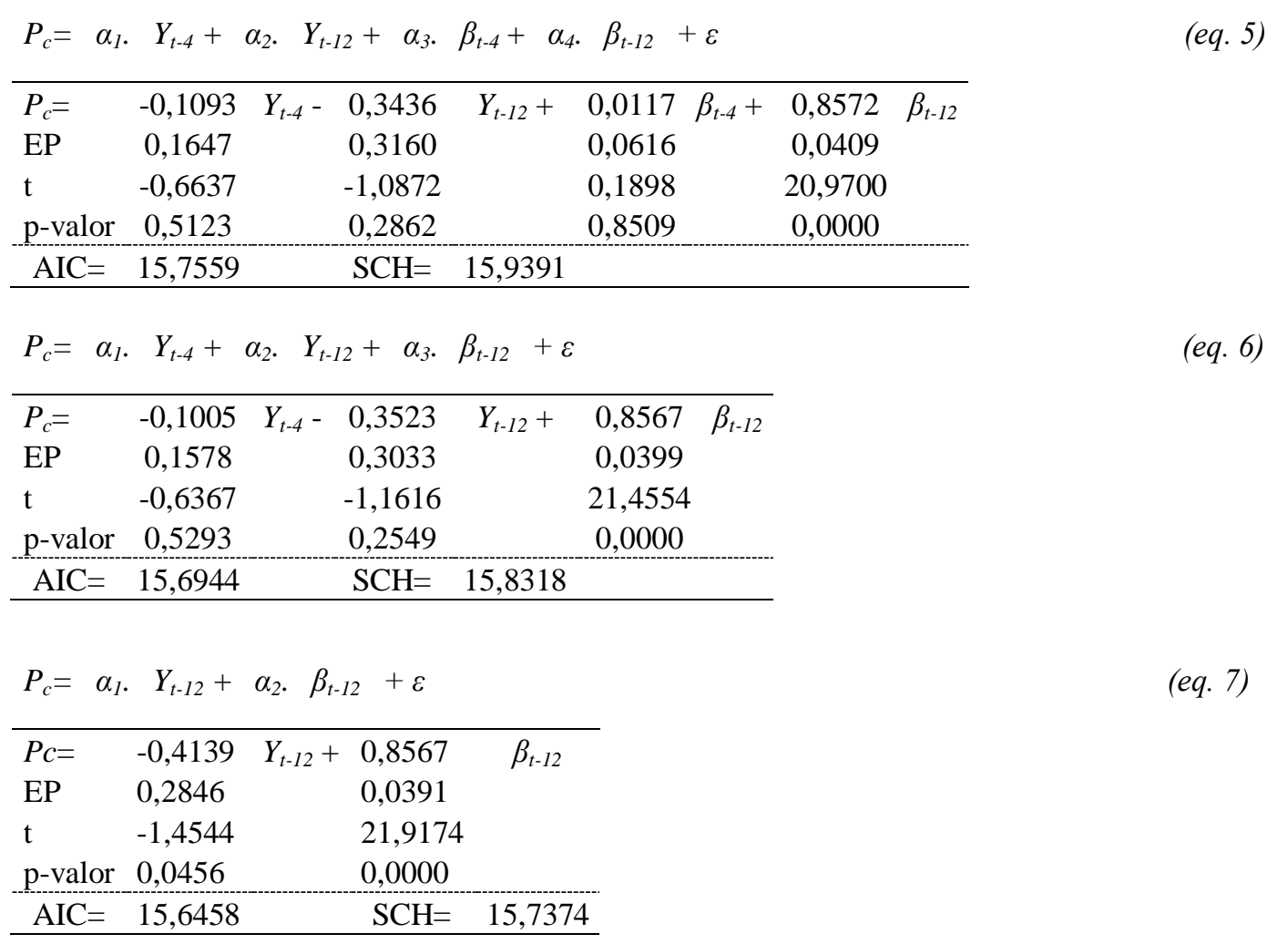

\section{Verificação}

$\mathrm{Na}$ verificação dos modelos escolhidos, avaliou-se que o comportamento médio dos seus resíduos foi satisfatório para o período utilizado no ajuste (1961-2005), obtendo-se resíduos próximos a um ruído branco, conforme figura 3. Pela tabela 2, pode-se afirmar que os modelos mostraram-se satisfatórios, ao serem confrontados os dados no período excluído do ajuste (2006 a 2009), com propósito da verificação. 


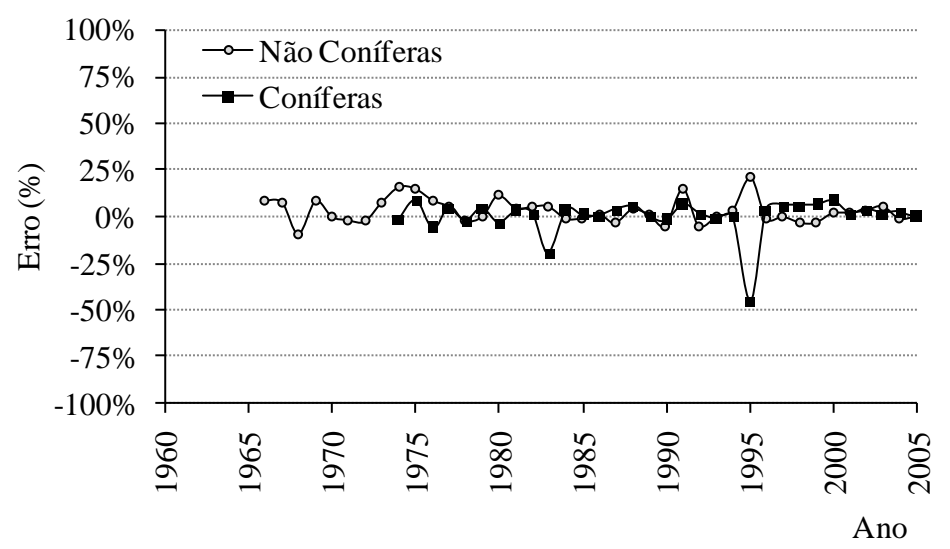

Figura 3. Erro percentual referente aos modelos $\operatorname{ARIMA}(2,1,1)$ para previsão da série de madeira de não coníferas e ARIMA $(1,1,1)$ para madeira de coníferas, entre os anos de 1961 e 2005.

Figure 3. Percentage error regarding the ARIMA $(2,1,1)$ to predict the non-coniferous wood series and ARIMA $(1,1,1)$ for coniferous wood, between the years 1961 and 2005.

Tabela 2. Estimativas da produção de madeira serrada entre os anos de 2006 a 2009, pelos modelos ARIMA $(2,1,1)$ referente à série de não coníferas e ARIMA $(1,1,1)$ para coníferas.

Table 2. Lumber production estimates from 2006 to 2009, by the ARIMA $(2,1,1)$ for a non-coniferous series and ARIMA $(1,1,1)$ for conifers.

\begin{tabular}{lccccc}
\hline & Modelo & Período & Valor real* & Valor estimado* & Diferença \\
\hline Não conífera & ARIMA $(2,1,1)$ & 2006 & 14.719 & 14.425 & $-2 \%$ \\
& & 2007 & 14.837 & 14.392 & $-3 \%$ \\
& 2008 & 16.110 & 16.916 & $5 \%$ \\
& & 2009 & 16.110 & 15.949 & $-1 \%$ \\
\hline Conífera & ARIMA $(1,1,1)$ & 2006 & 9.078 & 9.169 & $1 \%$ \\
& & 2007 & 9.577 & 10.535 & $10 \%$ \\
& & 2008 & 8.797 & 8.709 & $-1 \%$ \\
& & 2009 & 8.470 & 8.385 & $-1 \%$ \\
\hline
\end{tabular}

*(x $\left.1.000 \mathrm{~m}^{3}\right)$

Os coeficientes CDT encontrados pelos modelos sugerem um bom ajustamento e, consequentemente, melhores previsões para as séries analisadas (Tabela 3). Ressalte-se que esse coeficiente varia entre zero e um, sendo que zero indica um ajuste perfeito. Com relação a PT, PV e PC, o ideal é valor baixo para PT e PV e valor alto para PC (Castro et al., 2011), o que também foi observado nos resultados obtidos.

Tabela 3. Componentes de desigualdade de Theil para avaliação da previsão da produção anual de madeira serrada de não coníferas e coníferas no Brasil, no período de 1961 a 2009.

Table 3. Theil inequality components to assess the forecast annual production of non-coniferous and coniferous lumber in Brazil, from 1961 to 2009.

\begin{tabular}{lcc}
\hline Testes & Não coníferas & Coníferas \\
\hline Raiz do erro de previsão quadrático médio (REPQM) & 527,97 & 548,43 \\
Erro absoluto médio (EAM) & 363,54 & 346,94 \\
Percentual do erro absoluto médio (PEAM) & 4,50 & 5,12 \\
Coeficiente de desigualdade de Theil (CDT) & 0,02580 & 0,03535 \\
$\quad$ Proporção de tendenciosidade (PT) & 0,08649 & 0,01059 \\
$\quad$ Proporção de variância (PV) & 0,00451 & 0,00521 \\
$\quad$ Proporção de covariância (PC) & 0,90900 & 0,98420 \\
\hline
\end{tabular}


O período de tempo utilizado para ajuste do modelo (1961-2005) e os anos utilizados para validar o modelo (2006-2009) podem ser visualizados na figura 4, ao serem confrontados os dados observados e os estimados pelos respectivos modelos.
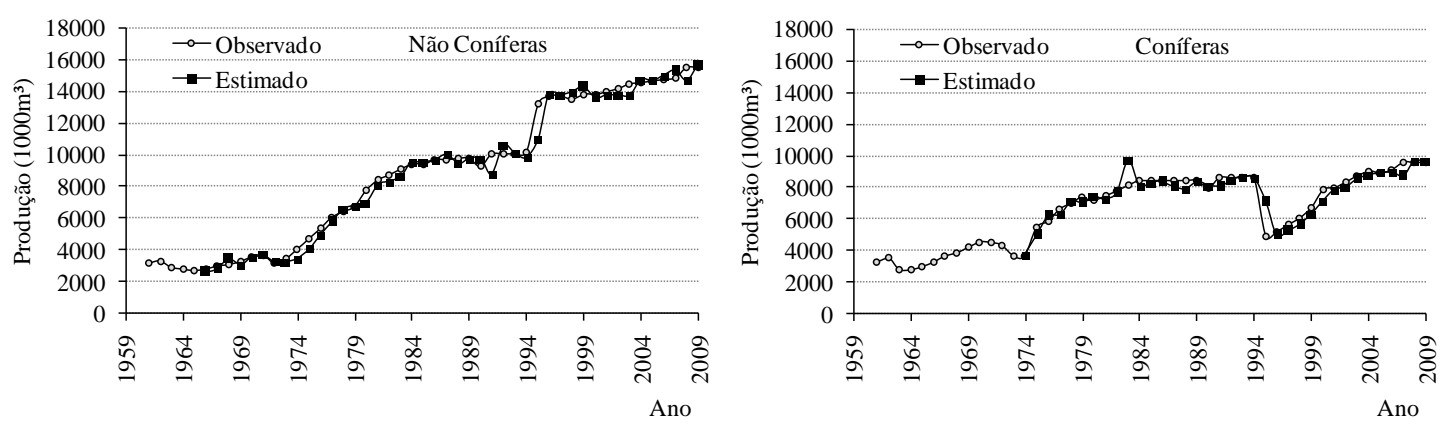

Figura 4. Valores observados e valores previstos segundo os modelos ARIMA $(2,1,1)$ e ARIMA $(1,1,1)$ para a produção anual de madeira serrada de não coníferas e coníferas no Brasil, respectivamente, no período de 1961 a 2009.

Figure 4. Observed and predicted values according to the ARIMA $(2,1,1)$ and ARIMA $(1,1,1)$ for the annual production of non-coniferous and coniferous sawn timber of non-coniferous and coniferous in Brazil, respectively, from 1961 to 2009.

De acordo com os ajustes, valores semelhantes entre os dados observados e previstos foram encontrados (Figura 4). Desse modo, pode-se dizer que os modelos de previsão foram adequados e podem ser empregados para prever a produção de madeiras de não coníferas e coníferas no Brasil.

Destaca-se a vantagem dos modelos ARIMA em prever a produção de produtos florestais em idades futuras sem precisar levar em conta as relações econômicas, por exemplo, que geraram as séries.

Em outros trabalhos, essa metodologia clássica de Box \& Jenkins foi aplicada com sucesso para fins de previsão de preços e de produção de matérias-primas de base florestal. Silva e Silva (1996); Coelho Júnior et al. (2006a, b) analisaram os preços de carvão vegetal em Minas Gerais, Brasil; Castro et al. (2011) avaliaram a produção de celulose de fibra curta para o Brasil; Cordeiro et al. (2010) realizaram a previsão do preço de exportação da madeira serrada de Pinus no Brasil.

\section{CONCLUSÕES}

- O modelo ARIMA $(2,1,1)$ foi eficiente e apresentou projeção satisfatória para a produção anual de madeira serrada de não coníferas, podendo ser utilizado para prever a produção futura em curtos prazos de forma confiável.

- O modelo ARIMA $(1,1,1)$ foi eficiente e apresentou projeção satisfatória para a produção anual de madeira serrada de coníferas.

\section{REFERÊNCIAS}

ALMEIDA, A. N.; ÂNGElO, H.; SILVA, J. C. G. L.; NUÑES, B. E. C. Análise econométrica do mercado de madeira em tora para o processamento mecânico no estado do Paraná. Scientia Forestalis, v. 37, n. 84, p. 377 - 386, 2009.

ASSOCIAÇÃO BRASILEIRA DE INDÚSTRIAS DO PROCESSAMENTO DA MADEIRA (ABIMCI).
$\begin{aligned} & \text { Programa Nacional de Qualidade da Madeira (PNQM). Disponível em: }\end{aligned}$ $<$ http://www.abimci.com.br/index.php?option=com_content\&view=category\&layout=blog\&id=14\&Itemi $\mathrm{d}=20$ > . Acesso em: 25 out. 2011.

BOX, G. E. P.; JENKINS, G. M. Time series analysis forecasting and control. San Francisco: HoldenDay, 1976. 375 p. 
CASTRO, A. F. N. M. Efeito da idade e de materiais genéticos de Eucalyptus sp. na madeira e carvão vegetal. 2011. 86 p. Dissertação (Mestrado em Ciência Florestal) - Universidade Federal de Viçosa, Viçosa, MG, 2011.

CASTRO, R. V. O.; MENDES, A. F. N.; ATAÍDE, G. da M.; COSTA, J. M. F. N.; VIEIRA, J. P. G.; SANTOS, R. C. dos; SOARES, C. P. B.; ARAÚJO JÚNIOR C. A. Projeções da produção de celulose de fibra curta no Brasil. Revista Floresta. v. 41, n. 2, p. 369 - 376, 2011.

COELHO JÚNIOR, L. M.; REZENDE, J. L. P.; CALEGARIO, N.; SILVA, M. L. Análise longitudinal dos preços do carvão vegetal no estado de Minas Gerais. Revista Árvore, v. 30, n. 3, p. 429 - 438, 2006 a.

COELHO JÚNIOR, L. M.; REZENDE, J. L. P.; SÁFADI, T.; CALEGARIO, N. Análise temporal do preço do carvão vegetal oriundo de floresta nativa e de floresta plantada. Scientia Forestalis, n. 70, p. 39 - 48, 2006b.

CORDEIRO, S. A.; SOARES, N. S.; BRAGA, M. J.; SILVA, M. L. Previsões do preço de exportação da madeira serrada de Pinus no Brasil. Scientia Forestalis, v. 38, n. 86, p. 205 - 214, 2010.

ECONOMETRIC EVIEWS. Eviews 5. Quantitative Micro Software, V5. 2004 (Software Econométrico).

ENDERS, W. Applied Econometric Time Series. New York: John Wiley, 1995. 433 p.

FOOD AND AGRICULTURE ORGANIZATION OF THE UNITED NATIONS (FAO). Disponível em: <http://faostat.fao.org/site/630/default.aspx>. Acesso em: 21 out. 2011.

GUJARATI, D. N. Econometria básica. 4. ed. São Paulo: Elsevier, 2006. 812 p.

MYNBAEV, T. K.; LEMOS, A. Manual de econometria. 2004, 348 p.

PEREZ, P. L.; BACHA, C. J. C. Evolução da produção e dos consumos interno e externo de madeira serrada do Brasil. Agroanalyses (FGV), v. 26, n. 8, 2006.

PONCE, R. H. Madeira serrada de eucalipto: desafios e perspectivas. In: SEMINÁRIO INTERNACIONAL DE UTILIZAÇÃO DA MADEIRA DE EUCALIPTO PARA SERRARIA, 1995, São Paulo. Anais... São Paulo: IPEF, IPT, IUFRO, LCF, ESALQ, USP, 1995. p. 50 - 58.

SEDDIGHI, H. R.; LAWYER, K. A.; KATOS, A. V. Econometrics - a pratical approach. London: Routledge, 2000. $396 \mathrm{p}$.

SILVA, M. L.; SILVA, J. M. A. Análise do comportamento temporal dos preços do carvão vegetal: aplicação do modelo e a avaliação da metodologia "Box and Jenkins". Revista Árvore, v. 20, n. 1, p. 57 67, 1996.

VITAL, M. Florestas independentes no Brasil. Rio de Janeiro: BNDES Setorial, n. 29, p. 77 - 130, 2009.

ZENID, G. J. Identificação e grupamento das madeiras serradas empregadas na construção civil habitacional na cidade de São Paulo. Piracicaba. 169 p. Dissertação (Mestrado) - Escola Superior de Agricultura Luiz de Queiroz, Universidade de São Paulo, 1997. 188 p. 
